\title{
Physical characterization of the Karin family ${ }^{\star}$
}

\author{
P. Vernazza ${ }^{1}$, M. Birlan ${ }^{2}$, A. Rossi ${ }^{3}$, E. Dotto ${ }^{4}$, D. Nesvorny ${ }^{5}$, R. Brunetto ${ }^{6}$, \\ S. Fornasier ${ }^{7}$, M. Fulchignoni ${ }^{1}$, and S. Renner ${ }^{1}$ \\ 1 LESIA, Observatoire de Paris, 92195 Meudon Principal Cedex, France \\ e-mail: pierre.vernazza@obspm. fr \\ 2 IMCCE, Observatoire de Paris, 77 Av. Denfert Rochereau, 75014 Paris Cedex, France \\ 3 Spaceflight Dynamics Section, ISTI-CNR, via Moruzzi, 1, 56124 Pisa, Italy \\ 4 INAF, Osservatorio Astronomico di Roma, via Frascatti 33, 00040 Monteporzio Catone (Roma), Italy \\ 5 Southwest Research Institute, 1050 Walnut St. Suite 400, Boulder, CO 80302, USA \\ 6 Dipartimento di Fisica, Universitá di Lecce, via Arnesano, 73100, Lecce, Italy \\ 7 CISAS-Astronomy Dep., Padova University, vicolo dell'Osservatorio, 2, 35122 Padova, Italy
}

Received 8 June 2006 / Accepted 8 September 2006

\section{ABSTRACT}

\begin{abstract}
Aims. The Karin cluster is a small asteroid family that formed $5.8 \pm 0.2 \mathrm{Myr}$ ago in the outer main belt. This is an exceptionally young age for an asteroid family. To investigate the composition and homogeneity of the members of this family, we started a spectroscopic survey in the visible and in the near-IR.

Methods. We observed 24 Karin asteroid members in the visible and 6 members in the near-IR.

Results. In the visible range, all the objects share the same characteristics: a maximum around $\lambda=0.75 \mu \mathrm{m}$, and a spectral slope spanning a continuous but limited range; in the NIR, our spectra show a similar behaviour. Our results suggest global homogeneity of the parent body and none of the investigated objects seems to be an interloper. These results are consistent with the dynamical hypothesis of a common origin. Finally, the range of spectral slopes is similar with the range of slopes for OC meteorites. We interpret this result as an indication of a low degree of spatial alteration for the observed surfaces. This result is coherent with the young age of the family.
\end{abstract}

Key words. methods: data analysis - techniques: spectroscopic - minor planets, asteroids

\section{Introduction}

Much of what we observe in the asteroid belt today is the result of collisions between asteroids. Remnants of a few tens of major collisional events have been recognized as clusters of objects in the orbital element space and referred to as "asteroid families". Zappala et al. (1995) made a systematic search for families in the known asteroid population based on orbital parameters analysis. All the families so far identified are relatively old (50 Myr $\leq$ age $\leq 3.5$ Gyrs). As a result the members of these families have undergone significant collisional and dynamical evolution since their formation (Marzari et al. 1999; Bottke et al. 2001; Nesvorný et al. 2002).

Physical characterization of members of several families, deduced by spectroscopic observations, showed similarities among asteroids belonging to the same family (Florczak et al. 1998; Doressoundiram et al. 1998; Lazzaro et al. 1999; Cellino et al. 2001). This is a strong observational confirmation of the common origin of these bodies.

Recently, Nesvorný \& Bottke (2004) have integrated backward in time the known 90 members of the 832 Karin family, a well defined cluster of asteroids in the proper elements space embedded within the larger Koronis family. The orbital elements of the Karin family members converge towards a single parent-body orbit $5.8 \pm 0.2 \mathrm{Myr}$ ago. This is the first time that

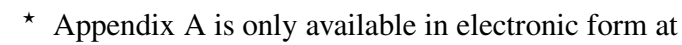
http: //www . aanda. org a collisional breakup event can be precisely dated with dynamical computations. The observed size distribution of the Karin family, as originally found by Nesvorný et al. (2002), included two large similarly sized bodies and a continuum of smaller objects ranging between 2 and $7 \mathrm{~km}$ in diameter. Later on, Nesvorný \& Bottke (2004) have found that the second largest body, $45071990 \mathrm{FV}$, is an interloper. With this correction, the size distribution of the Karin cluster is less unusual: one large body and a continuum of small ones that fits well into the size distribution produced by catastrophic disruptions. Recent simulations carried out by Nesvorný et al. (2006) took into account the unobserved sub-km fragments, which are believed to represent a large fraction of the parent body mass, and obtained a parent body of about $33 \mathrm{~km}$ in size. Michel et al. (2003, 2004) modeled the collision of the parent body of this family.

This discovery offers an excellent opportunity for physical studies of a young family whose members have apparently suffered limited dynamical and collisional erosion. A spectroscopic investigation of the family members allows us to:

i) have some hints on the internal structure and composition of the parent body: similar spectral features would indicate a possible homogeneous composition of the parent body, while some differences in behavior or wavelength position of the spectral features would give some information about a possible differentiated structure of the parent body;

ii) check differences or trends in the distribution of the spectral slopes, in order to investigate possible effect of a short time 
Table 1. Observational circumstances for the Karin members: the order is with respect to the observation date.

\begin{tabular}{|c|c|c|c|c|c|c|c|c|}
\hline Object & Day & UT & $\lambda(\mu \mathrm{m})$ & $\begin{array}{c}\text { CFHT } \\
\text { am }\end{array}$ & $V . \operatorname{mag}$ & $\alpha_{\mathrm{s}}$ & $R(\mathrm{AU})$ & $\Delta(\mathrm{AU})$ \\
\hline 832 & 02 Mar. 05 & $06: 46-07: 03$ & $0.45-0.95$ & 1.12 & 16.5 & 20.3 & 2.83 & 2.57 \\
\hline 69880 & 02 Mar. 05 & $07: 40-08: 40$ & $0.45-0.95$ & 1.01 & 19 & 14.7 & 2.82 & 2.06 \\
\hline 13765 & 02 Mar. 05 & $09: 21-09: 58$ & $0.45-0.95$ & 1.01 & 18.5 & 7.3 & 2.88 & 1.95 \\
\hline 13807 & 02 Mar. 05 & $10: 22-10: 59$ & $0.45-0.95$ & 1.02 & 18.2 & 4.8 & 3.08 & 2.12 \\
\hline 48369 & 02 Mar. 05 & $11: 54-12: 52$ & $0.45-0.95$ & 1.06 & 19.1 & 3.6 & 3.02 & 2.03 \\
\hline 79035 & 02 Mar. 05 & $13: 22-14: 22$ & $0.45-0.95$ & 1.22 & 19.4 & 7.9 & 3.08 & 2.14 \\
\hline 50715 & 02 Mar. 05 & $14: 33-15: 32$ & $0.45-0.95$ & 1.24 & 19.5 & 13 & 2.89 & 2.05 \\
\hline 7719 & 03 Mar. 05 & 06:05-07:05 & $0.45-0.95$ & 1.3 & 18.9 & 21 & 2.73 & 2.73 \\
\hline 832 & 03 Mar. 05 & $07: 29-07: 44$ & $0.45-0.95$ & 1.26 & 16.5 & 20.4 & 2.83 & 2.57 \\
\hline 103852 & 03 Mar. 05 & $12: 13-13: 37$ & $0.45-0.95$ & 1.18 & 19 & 2.0 & 2.83 & 1.85 \\
\hline 51089 & 03 Mar. 05 & $14: 00-15: 00$ & $0.45-0.95$ & 1.3 & 19.6 & 9.9 & 2.85 & 1.94 \\
\hline 832 & 04 Mar. 05 & $06: 10-06: 25$ & $0.45-0.95$ & 1.06 & 16.5 & 20.4 & 2.83 & 2.57 \\
\hline 75668 & 04 Mar. 05 & $06: 52-08: 00$ & $0.45-0.95$ & 1.04 & 19.8 & 10.8 & 3.0 & 2.13 \\
\hline \multirow[t]{2}{*}{76019} & 04 Mar. 05 & $10: 03-11: 12$ & $0.45-0.95$ & 1.02 & 19.8 & 1.9 & 2.83 & 1.84 \\
\hline & & & & NTT & & & & \\
\hline 11728 & 29 Jan. 06 & $01: 13-02: 14$ & $0.45-0.95$ & 1.66 & 19.2 & 16.8 & 2.91 & 2.29 \\
\hline 20095 & 29 Jan. 06 & $02: 47-03: 41$ & $0.45-0.95$ & 1.6 & 18.4 & 8.7 & 2.79 & 1.87 \\
\hline 20089 & 29 Jan. 06 & $04: 10-04: 50$ & $0.45-0.95$ & 1.48 & 19 & 3.6 & 2.92 & 1.95 \\
\hline 15649 & 30 Jan. 06 & $05: 49-06: 52$ & $0.45-0.95$ & 1.35 & 19 & 5.6 & 2.97 & 2.01 \\
\hline 23338 & 30 Jan. 06 & $07: 24-08: 40$ & $0.45-0.95$ & 1.55 & 19.3 & 6.2 & 2.83 & 1.88 \\
\hline 40510 & 31 Jan. 06 & $01: 54-02: 52$ & $0.45-0.95$ & 1.68 & 19.2 & 10.9 & 2.97 & 2.10 \\
\hline 94089 & 31 Jan. 06 & $03: 03-03: 40$ & $0.45-0.95$ & 1.55 & 18.1 & 6.4 & 2.80 & 1.85 \\
\hline 40789 & 31 Jan. 06 & 04:00-04:44 & $0.45-0.95$ & 1.42 & 19 & 1.6 & 3.01 & 2.03 \\
\hline 91706 & 31 Jan. 06 & 04:54-06:01 & $0.45-0.95$ & 1.46 & 18.7 & 1 & 2.82 & 1.84 \\
\hline 40782 & 31 Jan. 06 & $07: 25-08: 41$ & $0.45-0.95$ & 1.58 & 19.2 & 6 & 3.03 & 2.08 \\
\hline 40921 & 01 Feb. 06 & 04:04-05:07 & $0.45-0.95$ & 1.57 & 19 & 1.2 & 3.05 & 2.07 \\
\hline \multirow[t]{2}{*}{43032} & 01 Feb. 06 & $06: 05-06: 47$ & $0.45-0.95$ & 1.32 & 19.4 & 9.6 & 3.04 & 2.17 \\
\hline & & & & IRTF & & & & \\
\hline 832 & 04 Nov. 03 & $06: 00-06: 40$ & $0.8-2.5$ & 1.2 & 16.1 & 21.2 & 2.65 & 2.23 \\
\hline 69880 & 04 Nov. 03 & $10: 15-11: 20$ & $0.8-2.5$ & 1.26 & 18.5 & 10 & 2.73 & 1.83 \\
\hline 13765 & 04 Nov. 03 & $11: 50-12: 40$ & $0.8-2.5$ & 1.15 & 17.6 & 0.5 & 2.70 & 1.71 \\
\hline \multirow[t]{2}{*}{13807} & 04 Nov. 03 & $14: 10-15: 00$ & $0.8-2.5$ & 1.2 & 18.1 & 10 & 2.85 & 1.95 \\
\hline & & & & TNG & & & & \\
\hline 4507 & 29 Feb. 04 & 01:00-01:06 & $0.5-0.95$ & 1.1 & 16.25 & 11.6 & 2.83 & 1.97 \\
\hline 10783 & 30 Aug. 05 & $22: 30-23: 55$ & $0.8-2.5$ & 1.4 & 17.7 & 9 & 2.86 & 1.92 \\
\hline 57735 & 31 Aug. 05 & $00: 15-02: 20$ & $0.8-2.5$ & 1.35 & 18.6 & 2 & 2.77 & 1.76 \\
\hline
\end{tabular}

exposition to space weathering processes. The effect of space weathering processes has been already found in the spectra of asteroids belonging to dynamical families (Eos, Koronis, Flora, Eunomia): as an example, Florczak et al. (1998) carried out visible spectroscopy of 42 members of the Flora clan, and interpreted the obtained spread out of the visible spectral slopes as due to a space weathering $(\mathrm{Sw})$ process which altered the surface of older members. The precise dating of the fragmentation event allows us to put a time tag on the effects of space weathering;

iii) identify likely interlopers that can seriously affect the derived size distribution of the family and the reconstruction of the original fields of ejection velocity of the fragments (Cellino et al. 2002).

Here we present visible spectra of 24 objects belonging to the Karin cluster as well as near-infrared spectra $(0.8-2.5 \mu \mathrm{m})$ for 6 objects. These results are analysed and discussed.

\section{Observations and data reduction}

Observations of the Karin family members were performed both in the visible and in the near-IR with NTT (La Silla, Chile), CFHT (Mauna Kea, Hawaii), IRTF (Mauna Kea, Hawaii) and TNG (La Palma, Canary Island). The observational circumstances are presented in Table 1, which lists the date and time for each observation, the wavelength range, $V$ magnitude, airmass, the solar phase angle $\left(\alpha_{\mathrm{s}}\right)$, the heliocentric distance $(R)$ and the topocentric distance $(\Delta)$.

\subsection{Visible}

\section{CFHT}

We observed 13 objects with the CFHT during three consecutive nights in March 2005. We used the low resolution spectrograph MOS with the R150 grism in the $0.4-1.0 \mu \mathrm{m}$ wavelength range with a slit width of $1.5^{\prime \prime}$.

\section{NTT}

Visible spectra for 13 objects were collected in January 2006 during four consecutive nights at NTT with EMMI in low resolution spectroscopy mode. We used the Grism 1 $(0.4-1.0 \mu \mathrm{m})$ with a slit width of $1.5^{\prime \prime}$.

\section{TNG}

We obtained a spectrum for the object 4507 in February 2004 with the spectrograph Dolores and the grism LR-R (slit width of $\left.1.5^{\prime \prime}\right)$.

Standard techniques for visible spectroscopy reduction have been used in order to obtain the reflectance. We used the software MIDAS for the data reduction, applying the general procedures described in the following steps. An average bias was created 
Table 2. List of parameters discussed in the text.

\begin{tabular}{|c|c|c|c|c|c|c|}
\hline Object & Type & $\begin{array}{l}\text { Slope }\left(\mu \mathrm{m}^{-1}\right) \\
\pm 0.015 \mu \mathrm{m}^{-1}\end{array}$ & $\begin{array}{c}\text { Visible } \\
\operatorname{Max}(\mu \mathrm{m}) \\
\pm 0.020 \mu \mathrm{m}\end{array}$ & $H$ (mag) & $\begin{array}{c}D(\mathrm{~km}) \\
A=0.15\end{array}$ & $\begin{array}{c}D(\mathrm{~km}) \\
A=0.25\end{array}$ \\
\hline 832 & Sk & 0.411 & 0.747 & 11.18 & 20.1 & 15.6 \\
\hline 7719 & Sk & 0.166 & 0.744 & 14 & 5.5 & 4.3 \\
\hline 11728 & Sk & 0.326 & 0.760 & 14.2 & 5 & 3.9 \\
\hline 13765 & $\mathrm{Sq}$ & 0.044 & 0.730 & 14.2 & 5 & 3.9 \\
\hline 13807 & $\mathrm{Sq}$ & 0.249 & 0.745 & 13.7 & 6.3 & 4.9 \\
\hline 15649 & Sk & 0.265 & 0.757 & 14.6 & 4.2 & 3.2 \\
\hline 20089 & $\mathrm{~S}$ & 0.581 & 0.769 & 14.9 & 3.6 & 2.8 \\
\hline 20095 & $\mathrm{Sq}$ & 0.200 & 0.750 & 14.2 & 5 & 3.9 \\
\hline 23338 & Q & 0.101 & 0.740 & 15.1 & 3.3 & 2.6 \\
\hline 40510 & $S$ & 0.299 & 0.760 & 14.6 & 4.2 & 3.2 \\
\hline 40782 & $\mathrm{Sq}$ & 0.164 & 0.745 & 14.7 & 4.0 & 3.1 \\
\hline 40789 & $\mathrm{Sq}$ & 0.304 & 0.740 & 14.8 & 3.8 & 2.9 \\
\hline 40921 & $\mathrm{Sq}$ & 0.224 & 0.760 & 14.8 & 3.8 & 2.9 \\
\hline 43032 & Sk & 0.330 & 0.758 & 14.6 & 4.2 & 3.2 \\
\hline 48369 & $\mathrm{Sq}$ & 0.165 & 0.736 & 14.8 & 3.8 & 2.9 \\
\hline 50715 & Sk & 0.267 & 0.732 & 14.8 & 3.8 & 2.9 \\
\hline 51089 & S & 0.288 & 0.752 & 15.2 & 3.2 & 2.4 \\
\hline 69880 & $\mathrm{Sq}$ & 0.174 & 0.738 & 14.11 & 5.2 & 4.0 \\
\hline 75668 & Sk & 0.208 & 0.738 & 14.28 & 4.9 & 3.8 \\
\hline 76019 & $\mathrm{Sq}$ & 0.117 & 0.730 & 15.69 & 2.5 & 1.9 \\
\hline 79035 & $\mathrm{Sq}$ & 0.101 & 0.728 & 14.33 & 4.7 & 3.65 \\
\hline 91706 & $\mathrm{Sq}$ & 0.159 & 0.735 & 14.63 & 4.1 & 3.2 \\
\hline 94089 & $\mathrm{Sq}$ & 0.264 & 0.755 & 14.11 & 5.2 & 4.04 \\
\hline \multirow[t]{2}{*}{103852} & $S$ & 0.410 & 0.757 & 15.11 & 3.3 & 2.6 \\
\hline & $\begin{array}{c}\text { Band I }(\mu \mathrm{m}) \\
\pm 0.02 \mu \mathrm{m}\end{array}$ & Band II $(\mu \mathrm{m})$ & $\begin{array}{c}\text { Visble and Near-IR } \\
\text { BAR }\end{array}$ & $\begin{array}{l}\text { Slope }\left(\mu \mathrm{m}^{-1}\right) \\
\pm 0.015 \mu \mathrm{m}^{-1}\end{array}$ & & \\
\hline 832 & 0.92 & $2.01 \pm 0.04$ & $0.92 \pm 0.18$ & 0.077 & & \\
\hline 13765 & 0.94 & $2 \pm 0.05$ & $0.91 \pm 0.14$ & -0.071 & & \\
\hline 13807 & 0.95 & $2 \pm 0.06$ & $0.75 \pm 0.11$ & -0.065 & & \\
\hline 69880 & 0.96 & $2.03 \pm 0.07$ & $1.36 \pm 0.28$ & -0.006 & & \\
\hline
\end{tabular}

for each night and was subtracted to all our images. These images were divided by a normalized flatfield to remove pixel to pixel sensitivity variation on the CCD. At this point the twodimensional spectra were collapsed to one-dimension and then calibrated in wavelength. In order to perform a good correction for atmospheric extinction, we collected spectra for different solar analog stars at different airmasses during each night. We computed the ratio of these stars to estimate the difference in slope between these stars. The ratios normalized to unity were found to be almost flat with a maximum deviation of $3 \%$ from $0.55 \mu \mathrm{m}$ to $0.95 \mu \mathrm{m}$. Finally, we obtained the reflectance by dividing the asteroid spectrum by the solar analog's.

\subsection{Near-Infrared}

\section{IRTF}

An observing run in November 2003 was devoted to near-IR observations at IRTF. This run was remotely conducted from Meudon-France (Birlan et al. 2004; Vernazza et al. 2005). We observed 4 objects. The spectrograph SpeX, combined with the $0.8 \times 15$ arcsec slit (resolving power $R=100$ ), was used for acquisition of the spectra in the $0.8-2.5 \mu \mathrm{m}$ wavelength range.

\section{TNG}

Two spectra have been recorded in the near-infrared with the Telescopio Nazionale Galileo (TNG) at La Palma in August 2005. We used the Near-Infrared Camera Spectrometer (NICS) equipped with the AMICI prism, which provides a resolving

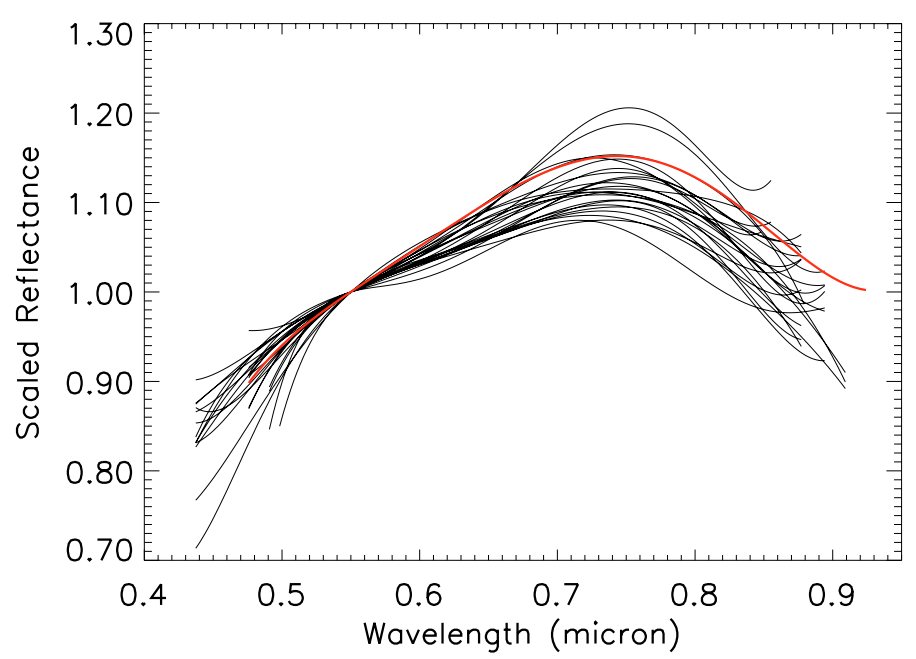

Fig. 1. The spectra of the 24 observed objects were fitted by a 6th order polynomial function and normalized to unity at $0.55 \mu \mathrm{m}$. The spectra are compatible with the presence of silicates. We can observe a color variation among the family members. In red, 832 Karin.

power of about 35 almost constant throughout the range $0.8-2.5 \mu \mathrm{m}$. We used a 1.5 -arcsec slit width.

The near-IR spectroscopy reduction was performed using the software Spextool 3.2 (Cushing et al. 2004), dedicated to reduce data obtained with Spex (IRTF), and with the software MIDAS for the reduction of the data obtained at TNG. In the near-IR, 
the technique for the data reduction is nearly identical to the one performed in the visible. The difference comes from the high luminosity and variability of the sky in the NIR. As a solution, the telescope was moved along the slit during the acquisition of the data in order to obtain a sequence of spectra located at two different positions (A and B) on the array. These paired observations provided near-simultaneous sky and bias measurements. A first step of the reduction process was to create bias and skysubtracted images A-B and B-A. The other steps included division by the flat-field image, extraction from two-dimensional images to one-dimensional arrays and the wavelength calibration of the spectra. After each collected spectrum, a star was observed very close in airmass. This strategy ensures a good correction for atmospheric extinction; this is very important, since we are looking for possible variation of the spectral slopes.

\section{Results}

\subsection{Visible data}

The visible data have been normalized to unity at $0.55 \mu \mathrm{m}$. The "raw" spectra are shown individually in the Appendix; the complete set of spectra has been fitted by 6th order polynomial functions and is shown in Fig. 1. All the observed objects belong to the S-complex. Our first analysis step has been to place the observed objects (Table 2) into the taxonomic system of Bus (1999).

Then, we made an analysis of each spectrum in terms of the position of the maximum and the spectral slope. In this wavelength range, the position of the maximum of the spectra is the only characteristic that allows us to investigate a possible variation of the surface composition. Longward of the maximum, all the Karin objects spectra exhibit the beginning of an absorption feature. This feature is most probably due to the presence of silicates like olivine and pyroxenes. Brunetto et al. (2006b) modeled the spectra of 832 Karin in the 0.4-2.5 micron range using the Shkuratov scattering model in order to constrain the possible surface composition of the asteroid and its space weathering degree. The space weathering was modeled with an exponential function following their experimental results. Brunetto et al. (2006b) found that olivine and orthopyroxene are the main surface components of 832 Karin (58.5\% and $38 \%$ respectively).

In the present study, the spectral coverage does not allow deeper investigation of the mineralogy. Compositionally induced changes in the position of the maxima occur with changes in the pyroxene to olivine proportion or with changes in their Fe or $\mathrm{Ca}$ content. For the observed objects, the maximum varies in the $0.728-0.77 \mu \mathrm{m}$ range (see Table 2 ). It should be noted that the slight variation in the location of the maximum is difficult to analyse in face of the error bars, which are mainly due to the noise and the proximity of the prominent atmospheric band at $0.7619 \mu \mathrm{m}$. If this variation is real, it could imply diversity in the surface composition of the family members. Alternatively, the family members could have a unique composition, but in this case the differences in the spectra would need to be explained by some other process, such as space weathering.

Indeed, laboratory experiments have shown that a planetary surface exposed to the effect of solar wind ion irradiation, cosmic rays and interplanetary dust bombardment, changes in color and spectral characteristics. The effect is a progressive darkening of the surfaces, and a reddening of the reflectance spectra in the UV-Vis-NIR range (Hapke 2001; Sasaki et al. 2001; Brunetto \& Strazzulla 2005; Brunetto et al. 2006a). These effects have been studied in order to obtain a link between the spectral properties of meteorites and the remote sensing data of asteroids. Actually, space weathering can explain the spectral mismatch between the most populous class of meteorites (ordinary chondrites, OC) and the surface spectra of their presumed (S-type) asteroidal parent bodies (Pieters et al. 2000; Adams \& McCord 1971; Strazzulla et al. 2005; Marchi et al. 2005). Using laser ablation experiments, Sasaki et al. (2001) and Brunetto et al. (2006a) estimated that micrometeoroid impacts may produce significant space weathering effects on a timescale of about $10^{8}$ years. Moreover, it has been shown recently that solar wind ion irradiation dominates asteroidal space weathering with a timescale to be of the order of $10^{4}-10^{6}$ years (Vernazza et al. 2006).

It is now widely accepted that the effects of spatial alteration can be investigated by measuring the spectral slope. We determined the spectral slopes in the $0.52-0.92 \mu \mathrm{m}$ range. The results are reported in Table 2 . The error bar does not take in account the solar analog error (3\%). For 23 objects, the slopes values are in the range $[0.04 ; 0.41] \mu \mathrm{m}^{-1}, 20089$ being an exception with a slope equal to 0.58 . Lazzarin et al. (2005) calculated the spectral slopes of a large sample (300) of OCs in the same wavelength range. As in their case, we consider the OC meteorites as the best analogues for S-type asteroids (the limitations of this assumption are discussed by Brunetto et al. 2006b). They found that $95 \%$ of OCs are below a slope of $0.208 \mu \mathrm{m}^{-1}$. They assumed this last value of slope to be an indicator for the space weathering process. Moreover, they found that many NEOs are redder (greater slope) than OCs, showing that space weathering may alter also the surface spectral properties of small size asteroids, usually considered little weathered. In the present work, 10 objects $(40 \%)$ have a slope value below the mentioned ( $\mathrm{Sw}$ ) limit, which makes them from this point of view compatible with OCs. The other objects have a slope greater than this limit, which may indicate the presence of $\mathrm{Sw}$ processes. Also, the average slope for the Karin family is $0.242 \pm 0.03$ if we include the object 20089 (the error being the error on the average, not the standard deviation), or $0.228 \pm 0.03$ if we exclude 20089 . These values are above the 0.208 limit of Lazzarin et al. (2005), and also above the average OCs' slope which is slightly lower than 0.100 . So, the Karin family spectra are on average a little bit redder than OCs, and this is a confirmation that the space weathering level is very low, but is still present and measurable. Jedicke et al. (2004) obtained asteroid colors from the Sloan Digital Sky Survey Moving Object Catalog for twelve asteroidal families belonging to the Scomplex (Koronis and Karin in particular). They found that the colours (PC1) of the Karin members are in the 0.32-0.4 range while the mean $\mathrm{PC} 1$ value for $\mathrm{OC}$ meteorites is $0.36 \pm 0.06$ (without the LL3 subclass - with PC1 $\approx 0.59$ ). As in our case, some of the Karin members are on average redder than OCs. Our results are in agreement with their findings.

The spectroscopic investigation of a family usually allows detection of the presence of interlopers. Interlopers can be identified when they are characterized by spectroscopic properties incompatible with those of their family. For instance, a C-type object belonging to a family of S-type members should be considered as a very likely interloper. In the present study, no object is genetically incompatible with the rest of the family.

We have also investigated the effect of the diameter. Using the absolute magnitude of each object and assuming an albedo equal to 0.15 or 0.25 (Table 2), Nesvorný \& Bottke (2004) calculated the diameters for all the family members. We did not find a relation between the slope and asteroid diameters, but this result can be attributed more to the very limited span of diameters present in the Karin clan than to some real physical property. 


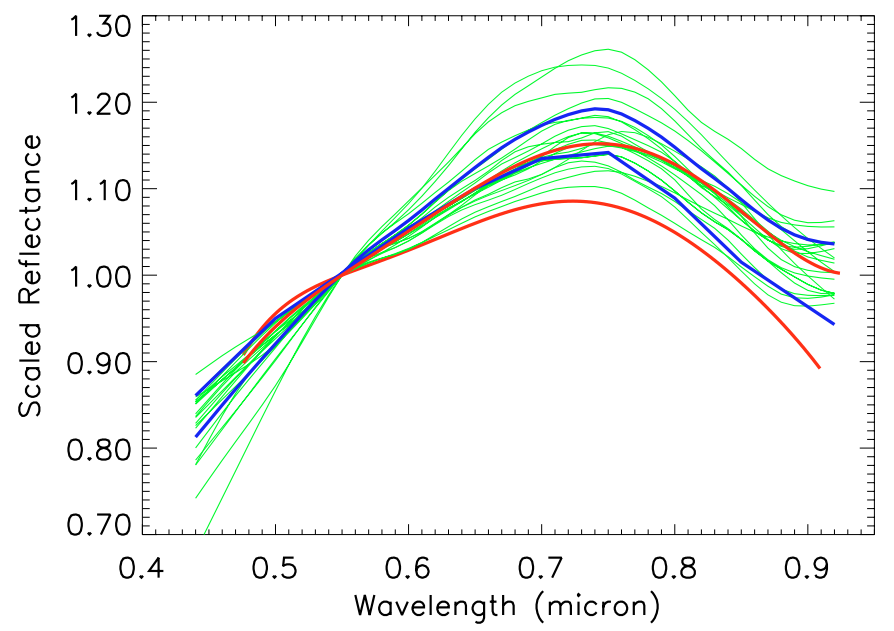

Fig. 2. The Karin cluster has been identified within the Koronis family. Here we show all the spectra for the Koronis family members (in green) obtained from the SMASS database. In red, the domain representing 92\% of the Karin members spectra. In blue, from bottom to top: the mean Sq type spectrum and the mean $\mathrm{S}$ type spectrum (Bus \& Binzel 2002a,b). The colors of the Karin members are less "red" than those of the Koronis ones. This result is in agreement with the respective age of each family (i.e. younger exposure ages for the surfaces of the Karin members).

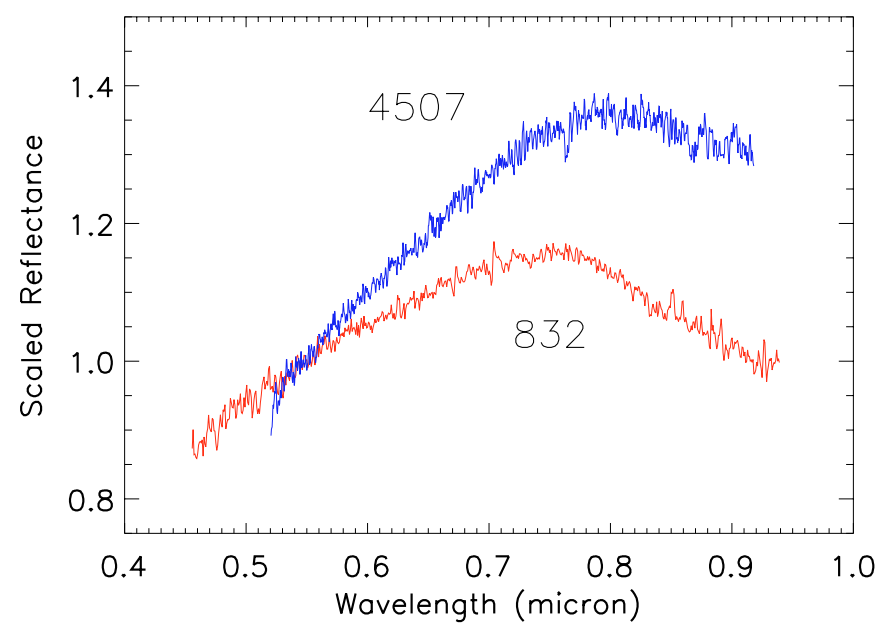

Fig. 3. We show the spectrum of 832 Karin and of the interloper 4507 (Nesvorný \& Bottke 2004). Both objects belong to the S-complex, but have very different spectral slopes and a different position of the maximum of their spectra.

We also observed the asteroid 4507, previously believed to belong to the Karin cluster (Nesvorný et al. 2002). As shown in Fig. 3, its spectrum is very different from the Karin one. First, its slope is much redder; second, the position of its maximum is located at greater wavelength $(0.82 \mu \mathrm{m}$ for $4507,0.75 \mu \mathrm{m}$ for 832 Karin) suggesting a different surface composition. Using a refined method to identify members of the Karin cluster, Nesvorný \& Bottke (2004) found that 4507 is an interloper. Our observations (Fig. 3) tend to confirm this result.

Finally, we compared the visible spectra of the Karin family with those of the Koronis clan (Fig. 2). The Koronis family is one of the largest and most well-defined families, first identified by Hirayama in 1918 (Hirayama 1918). The Karin parent body actually belonged to the older Koronis family (2.5-3 Gyr old). Binzel et al. (1993) observed 8 Koronis members (SMASS) and detected small spectral variations indicative of a modest

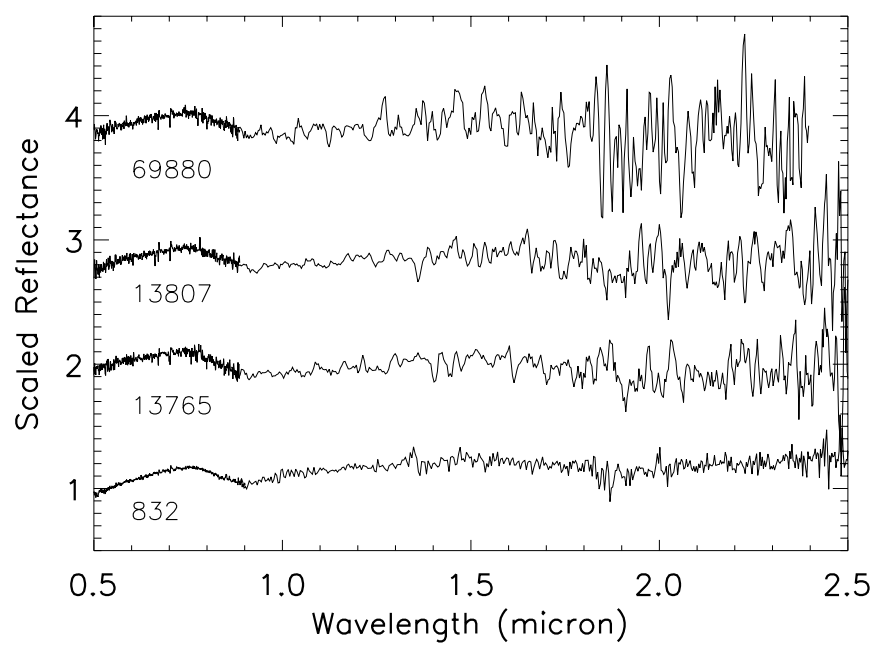

Fig. 4. Spectra of $832,13765,13807$, and 69880 in the $0.5-2.5 \mu \mathrm{m}$ range. The spectra were vertically displaced for clarity. The NIR spectra have been brought to lower resolution by Gaussian smoothing. The Gaussian width used was 4 points.

degree of differentiation of the parent body. Our results are consistent with those of Binzel et al. (1993). Moreover, we notice that the dispersion of the spectral slopes is much greater for the Koronis members than for the Karin objects. This may be explained by the collisional erosion undergone by the Koronis members (resurfacing through collisions).

Previous physical studies (Florczak et al. 1998; Doressoundiram et al. 1998; Lazzaro et al. 1999; Cellino et al. 2001) of asteroid families were based on visible spectroscopy. This spectral range allowed to derive substantial conclusions on the composition of the parent body. Indeed, our results based on the visible data suggest global homogeneity of the parent body. However, near-infrared observations of the family members may strengthen this first conclusion.

\subsection{Near-IR data}

We obtained spectra for 6 Karin cluster members in the $0.8-$ $2.5 \mu \mathrm{m}$ range. The data have been normalized to unity at $0.9 \mu \mathrm{m}$. The spectra are shown individually in the Appendix. The spectra have been brought to lower resolution by Gaussian smoothing. The Gaussian width used was 4 points. Four of those six objects have been observed in the visible (Table 1). As in the visible range, the 6 NIR spectra look very similar. They have a band minimum around $0.92 \mu \mathrm{m}$ and a maximum of the band around $1.5 \mu \mathrm{m}$.

We analyzed the spectra of $832,13765,13807$, and 69880 in terms of the spectral parametrization developed by Cloutis et al. (1986). For each spectrum, we computed the slope of continuum across the $1 \mu \mathrm{m}$ band, the areas and position of the absorption band centers located near 1 and $2 \mu \mathrm{m}$, which from now on will be referred to as BI and BII, respectively. The slope is representative of space weathering alteration while the other parameters are representative of the mineralogical composition.

First, we scaled the NIR spectra of $832,13765,13807$, and 69880 to the visible part using the overlap in the $0.8-0.9 \mu \mathrm{m}$ region (Fig. 4). Second, we defined the three maxima near 0.75 , 1.55 and $2.45 \mu \mathrm{m}$. The linear continuum for BI is given by the straight line tangent to the maxima near 0.75 and $1.55 \mu \mathrm{m}$, while for BII the continuum is determined by the maxima around 1.55 and $2.45 \mu \mathrm{m}$. For each band, the continuum was removed by 
dividing each band by the linear continuum in each corresponding region. The center of each band was computed after the continuum removal, using polynomial fits around the region of each reflectance minimum, with orders varying from 3 to 6 . The adopted values for band centers correspond to the polynomial that yields the best fit, and the uncertainty in band center position is given by the scatter due to the use of different polynomials and to the noise. The areas of BI and BII are also measured after removing the continua, and the parameter BAR is defined as the ratio between the areas of BII and BI. The spectral parameters for each spectrum are presented in Table 2.

The spectra are very noisy after $1.6 \mu \mathrm{m}$, and this explains the large error bar for the BAR value and the center of BII. Like in the visible, where we observed a variation of the location of the maximum, we obtain here a similar result with a slight variation of the band center position. Again, the noise certainly affects the calculation. Our results, including the global shape of the spectra as well as the values for the different parameters, suggest a similar mineralogical composition for the four objects but a slight variation of the composition in terms of pyroxene to olivine proportion and/or changes in their Fe or Ca content cannot be excluded. For the 4 objects, the weighted average for the BAR value is about $0.92 \pm 0.12$ which corresponds to a content of $44 \pm 8 \%$ orthopyroxene and $56 \pm 8 \%$ olivine (Cloutis et al. 1986; Gaffey et al. 1993). This composition is in agreement with the one found by Brunetto et al. (2006b) for the asteroid 832 Karin using the Shkuratov scattering model. Also, the avergage BAR value along the BI center values, places the objects in the loci of OC meteorites (BAR $\times$ BI center diagram) following the scheme of Gaffey et al. (1993).

Finally, we calculated the spectral slope for each composite spectrum and compared the values with those calculated by (Marchi et al. 2005) for Ordinary Chondrites. Marchi et al. (2005) chose a huge number of OC meteorites spectra from RELAB, considering only those meteorites for which no trace of alteration was reported. They calculated the slope of the continuum across the 1-micron absorption band for 184 OC spectra. They found that $95 \%$ of the slopes are in the $[-0.2$; $0.138] \mu \mathrm{m}^{-1}$ range, and they defined a $95 \%$ OC slope limit (slope $=0.138 \mu \mathrm{m}^{-1}$ ) beyond which an asteroid can be considered as "weathered". They found that about $94 \%$ of the MBAs have a slope higher than this limit.

Comparing the slopes of our spectra with those of OCs (Table 2) we find that they fall exactly in the OC range. In Fig. 5, we compare the 4 composite spectra with three OC spectra whose slopes are representative of the slope domain of OCs. The meteorite spectra are from the RELAB spectral database (http: //www . planetary. brown . edu/relab/). The Karin family spectra appear in the upper part of the OCs "domain" (i.e. slope). Also their band depth appears shallower than that of OC meteorites. Both aspects tend to confirm that the optical properties of the observed surfaces have undergone some modification in the last 5.8 Myr due to the $\mathrm{Sw}$ processes.

\section{Discussion}

\subsection{Dispersion of the slopes}

On the basis of the analysis of the visible and NIR spectra, our results suggest that the parent body of the Karin cluster was globally homogeneous. However, we note a quite "important" dispersion for the spectral slopes (in the visible). We will try to address this question and list the different processes able to explain this difference in slope.

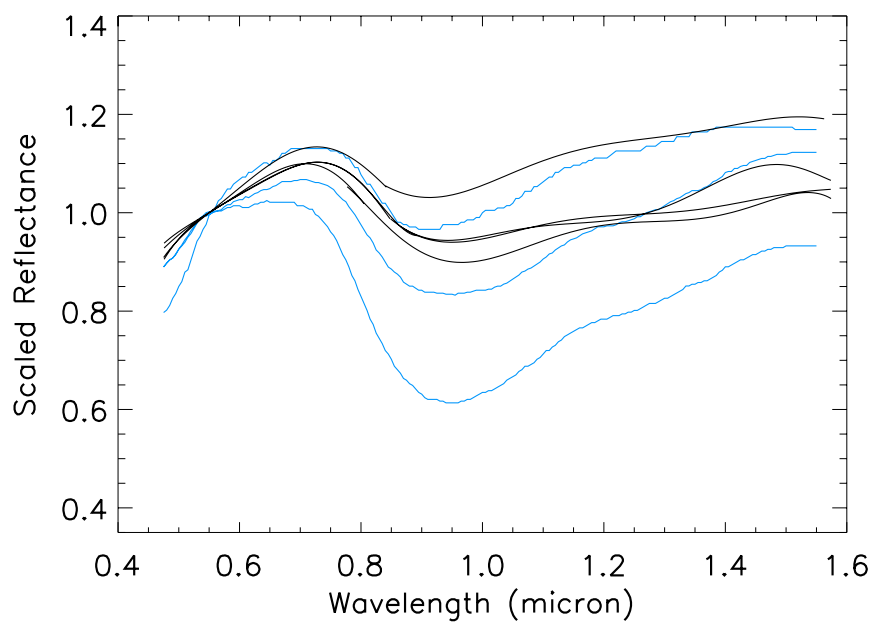

Fig. 5. In black, we show the spectra of 832 Karin, 13765, 13807, and 69880. We fitted the composite spectrum of these four objects using a 6 th order polynomial function. Since the spectra of 13765,13807 , and 69880 are dominated by the noise after $1.6 \mu \mathrm{m}$, we limited the fit to the $0.4-1.6 \mu \mathrm{m}$ range. In blue, we plot also the spectra of three ordinary chondrites (from bottom to the top: Aumale (L6), Jelica (LL6) and Mezoe-Madaras (L3)).

1) First, the observed dispersion may be due to a difference of the Sw degree. Considering that the surface age for all the observed surfaces might be the same, we could expect an almost negligible difference for the spectral slopes (same SW degree).

Indeed, while space weathering processes alter the optical properties of the uppermost thin layer of regolith, other processes such as the impact blanketing are competing with the above effects to periodically uncover optically unaltered material (Nesvorný et al. 2005). Consequently, the resulting surface of an asteroid is a complex product of these processes. In the hypothesis that the surfaces have undergone "the same amount of spatial alteration", the degree of the rejuvinating processes must have been slightly different for all objects. In this regard, the range of slopes that we observe, may stress the relevance of the refreshing processes (which are random).

2) Another reason that could be proposed, is that some of the objects were part of the crust of the parent body and that they kept their previous altered surface intact. Consequently, the difference in spectral slope observed within the family would be due to the former location of each object inside the parent body (i.e. interior or crust). However, it is not clear how some objects could have preserved a surface "older" than the age of the family. According to the recent results of Richardson et al. (2004), impact-induced seismic shaking can produce considerably regolith movements. This should have been quite important for the impact that formed the family, and presumably affected the surface of all the objects. We would expect that after the major collision that created the family, the surfaces of all the members were "reset". This would bring us back to the first explanation.

3) Third, the slope (especially in the visible) is not exactly proportional to the space weathering, since also scattering properties of the surface can affect it. As a result, it is true that the slope increases with the exposure, but even in the laboratory experiments a spread is observed (Brunetto \& Strazzulla 2005). From laboratory experiments, it is clear that the slope 
increases with the damage, but there is an intrinsic spread as well, even for very similar samples.

4) A last possible explanation is that, for a non spherical body, changes in the viewing aspect (e.g., edge-on versus end-on) can produce spectral slope changes (e.g., Gradie \& Veverka 1981).

At this point, we are unable to define which of the above explanations is the right one (if there is only one). However, it is interesting to note that all the families investigated until now, show a dispersion of the spectral slopes as well. In the case of a very old family like Koronis, the dispersion is even greater and in that case, the most plausible explanation might be the influence of the refreshing processes on the color.

\subsection{First $O C$-like $M B A s$ ?}

It is interesting to note that $40 \%$ of the observed objects (in the visible) are more Q- or OC-like (in terms of slope; see also Fig. 5). Q-type asteroids have been found among the NEO population (about $6 \%$ of $\approx 300$ surveyed NEOs are Q-types; see Binzel et al. (2002) for a recent review). In contrast, no Q-type asteroid has been found to date among the 2000 surveyed main belt asteroids (MBAs; e.g., Bus \& Binzel 2002a,b). This lack of spectrophotometric main-belt analogs for the OC meteorites is a long-debated and fundamental problem. The canonical interpretation of these results is that the lack of Q-type asteroids among the observationally sampled MBAs is related to asteroid-sizedependent effects on surface regolith or to the shorter collisional lifetimes of smaller asteroids (Binzel et al. 2002). Indeed, current spectrophotometric surveys of MBAs are largely incomplete in the size range of typical NEOs ( $\leq 5-\mathrm{km}$ diameters). Researchers hypothesized that: (i) survival lifetimes against catastrophic disruption (Davis et al. 2002) decrease with decreasing size. Thus, on average, as we examine smaller and smaller objects, we should see younger and younger surfaces; (ii) surfaces showing Q-type spectral properties should thus exist, on average, only among the smallest asteroids, which become easy spectroscopic targets only when they enter into NEO space; (iii) large, OClike asteroids in the main belt should show, on average, "spaceweathered" spectral properties, explaining why they are taxonomically classified as S-type asteroids (Nesvorný et al. 2005).

All the objects besides 832 Karin are in the size range of NEAs $(D \leq 6-\mathrm{km})$. A part of them $(40 \%)$ verifies the hypothesis suggested by the standard scenario, with a spectral slope being in the range of OCs slopes. But the rest of the family (60\%) begins to show a clear evidence of space weathering.

Here we have learned that an exposure age of 5.8 Myr starts to transform an OC spectrum in an S-type spectrum in a distinct way (true for $60 \%$ of objects). In the near future, other recent break-up events will be identified, at probably different heliocentric distances. Along our result, which stresses the efficiency of the $\mathrm{Sw}$ processes at $2.9 \mathrm{AU}$ in $5.8 \mathrm{Myr}$, we will be able to estimate the relative importance of various parameters (Sun-Object distance, surface age, composition) on the color of an asteroid.

\section{Conclusions}

Twenty-four spectra of the Karin cluster asteroids were obtained in the visible as well as six spectra in the near-IR.

In both wavelength ranges, the spectra show similar behavior, indicative of a similar composition and space weathering degree. None of the objects can be considered as an interloper, based on the obtained spectra, confirming the dynamical indication of a probable common origin from the fragmentation of a parent-body. Our results suggest global homogeneity of the parent body.

Comparing the spectral slope of the Karin members with the slope domain of ordinary chondrites, we found that the Karin members are slightly redder than OCs. We interpret this result as an indication of a low degree of spatial alteration for the surfaces of these objects, which is in agreement with the young age of the Karin family (5.8 Myr).

This spectroscopic investigation has shown that in the case of a young family, the dynamical indentification is very reliable.

Acknowledgements. The article is based on observations acquired with IRTF, TNG, CFHT and NTT telescopes, and the CODAM remote facilities. We thank all the telescope operators for their contribution. This research used spectra acquired by Hiroi, Pieters, and Gaffey, with the NASA RELAB facility at Brown University. We thank the anonymous referee for his pertinent and constructive remarks.

\section{References}

Adams, J. B., \& McCord, T. B. 1971, Science, 171, 567

Binzel, R. P., Lupishko, D., di Martino, M., Whiteley, R. J., \& Hahn, G. J. 2002, Asteroids III, 255

Binzel, R. P., Xu, S., \& Bus, S. J. 1993, Icarus, 106, 608

Birlan, M., Barucci, M. A., Vernazza, P., et al. 2004, New Astronomy, 9, 343

Bottke, W. F., Vokrouhlický, D., Brož, M., Nesvorný, D., \& Morbidelli, A. 2001, Science, 294, 1693

Brunetto, R., \& Strazzulla, G. 2005, Icarus, 179, 265

Brunetto, R., Romano, F., Blanco, A., et al. 2006a, Icarus, 180, 546 Brunetto, R., Vernazza, P., Marchi, S., et al. 2006b, Icarus, In press Bus, S. J. 1999, Ph.D. Thesis

Bus, S. J., \& Binzel, R. P. 2002a, Icarus, 158, 146

Bus, S. J., \& Binzel, R. P. 2002b, Icarus, 158, 106

Cellino, A., Zappalà, V., Doressoundiram, A., et al. 2001, Icarus, 152, 225

Cellino, A., Bus, S. J., Doressoundiram, A., \& Lazzaro, D. 2002, Asteroids III, 633

Cloutis, E. A., Gaffey, M. J., Jackowski, T. L., \& Reed, K. L. 1986, J. Geophys. Res., 91, 11641

Cushing, M. C., Vacca, W. D., \& Rayner, J. T. 2004, PASP, 116, 362

Davis, D. R., Durda, D. D., Marzari, F., Campo Bagatin, A., \& Gil-Hutton, R. 2002, Asteroids III, 545

Doressoundiram, A., Barucci, M. A., Fulchignoni, M., \& Florczak, M. 1998, Icarus, 131, 15

Florczak, M., Barucci, M. A., Doressoundiram, A., et al. 1998, Icarus, 133, 233 Gaffey, M. J., Burbine, T. H., Piatek, J. L., et al. 1993, Icarus, 106, 573

Gradie, J., \& Veverka, J. 1981, in Lunar and Planetary Institute Conference Abstracts, 359

Hapke, B. 2001, J. Geophys. Res., 106, 10039

Hirayama, K. 1918, AJ, 31, 185

Jedicke, R., Nesvorný, D., Whiteley, R., Ivezić, Ž., \& Jurić, M. 2004, Nature, 429,275

Lazzarin, M., Marchi, S., Magrin, S., \& Licandro, J. 2005, MNRAS, 359, 1575

Lazzaro, D., Mothé-Diniz, T., Carvano, J. M., et al. 1999, Icarus, 142, 445

Marchi, S., Brunetto, R., Magrin, S., Lazzarin, M., \& Gandolfi, D. 2005, A\&A, 443, 769

Marzari, F., Farinella, P., \& Davis, D. R. 1999, Icarus, 142, 63

Michel, P., Benz, W., \& Richardson, D. C. 2003, Nature, 421, 608

Michel, P., Benz, W., \& Richardson, D. C. 2004, Icarus, 168, 420

Nesvorný, D., \& Bottke, W. F. 2004, Icarus, 170, 324

Nesvorný, D., Bottke, W. F., Dones, L., \& Levison, H. F. 2002, Nature, 417, 720

Nesvorný, D., Jedicke, R., Whiteley, R. J., \& Ivezić, Ž. 2005, Icarus, 173, 132

Nesvorný, D., Enke, B. L., Bottke, W. F., et al. 2006, Icarus, In press

Pieters, C. M., Taylor, L. A., Noble, S. K., et al. 2000, Meteoritics and Planetary Science, 35, 1101

Richardson, J. E., Melosh, H. J., \& Greenberg, R. 2004, Science, 306, 1526

Sasaki, S., Nakamura, K., Hamabe, Y., Kurahashi, E., \& Hiroi, T. 2001, Nature, 410,555

Strazzulla, G., Dotto, E., Binzel, R., et al. 2005, Icarus, 174, 31

Vernazza, P., Mothé-Diniz, T., Barucci, M. A., et al. 2005, A\&A, 436, 1113

Vernazza, P., Brunetto, R., Strazzulla, G., et al. 2006, A\&A, 451, L43

Zappala, V., Bendjoya, P., Cellino, A., Farinella, P., \& Froeschle, C. 1995, Icarus, 116, 291 
P. Vernazza et al.: Karin family, Online Material $p$ l

\section{Online Material}


P. Vernazza et al.: Karin family, Online Material p 2

6. Appendix: Relative Reflectance for the Karin family asteroids 
P. Vernazza et al.: Karin family, Online Material p 3
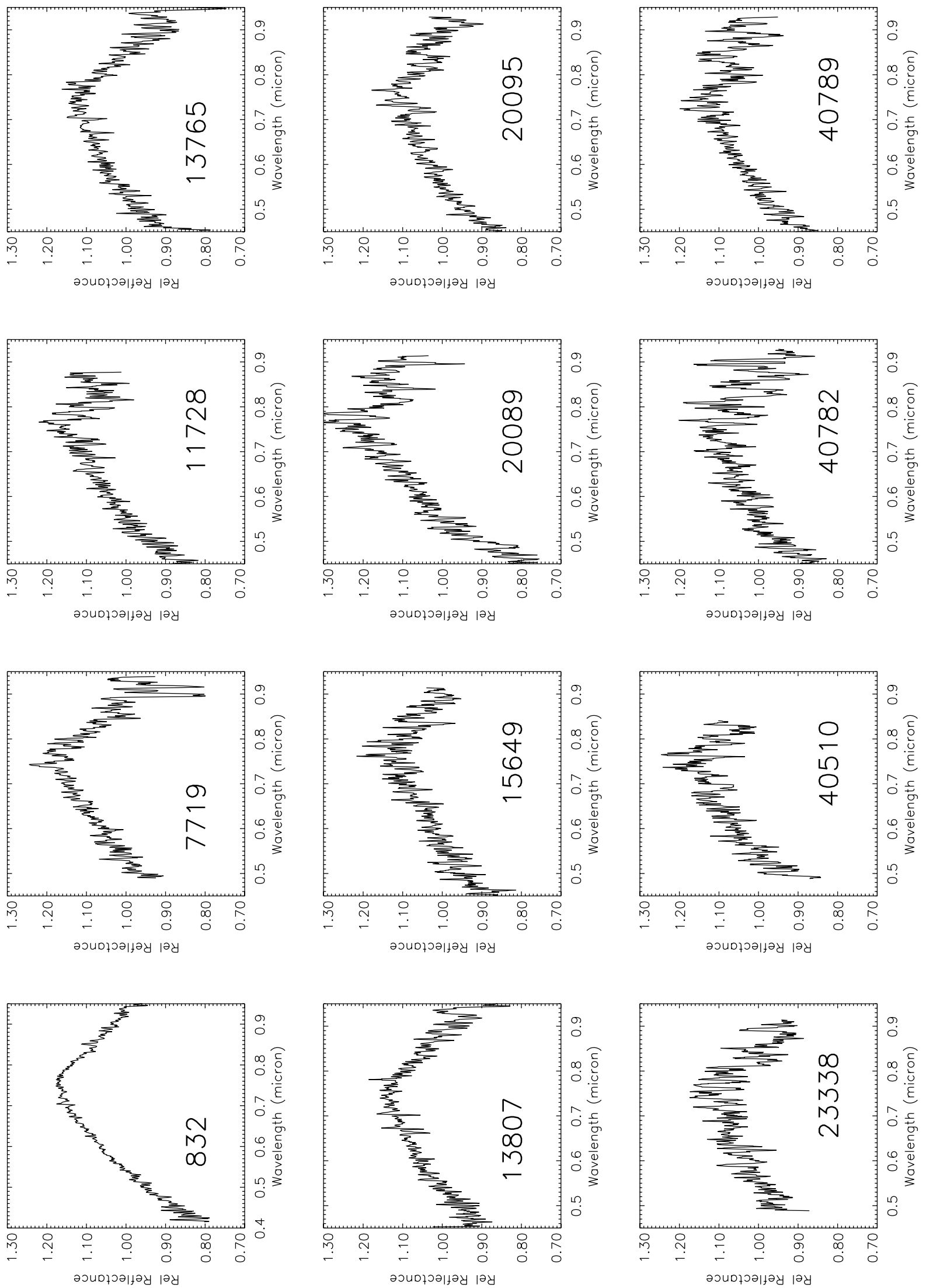
P. Vernazza et al.: Karin family, Online Material p 4
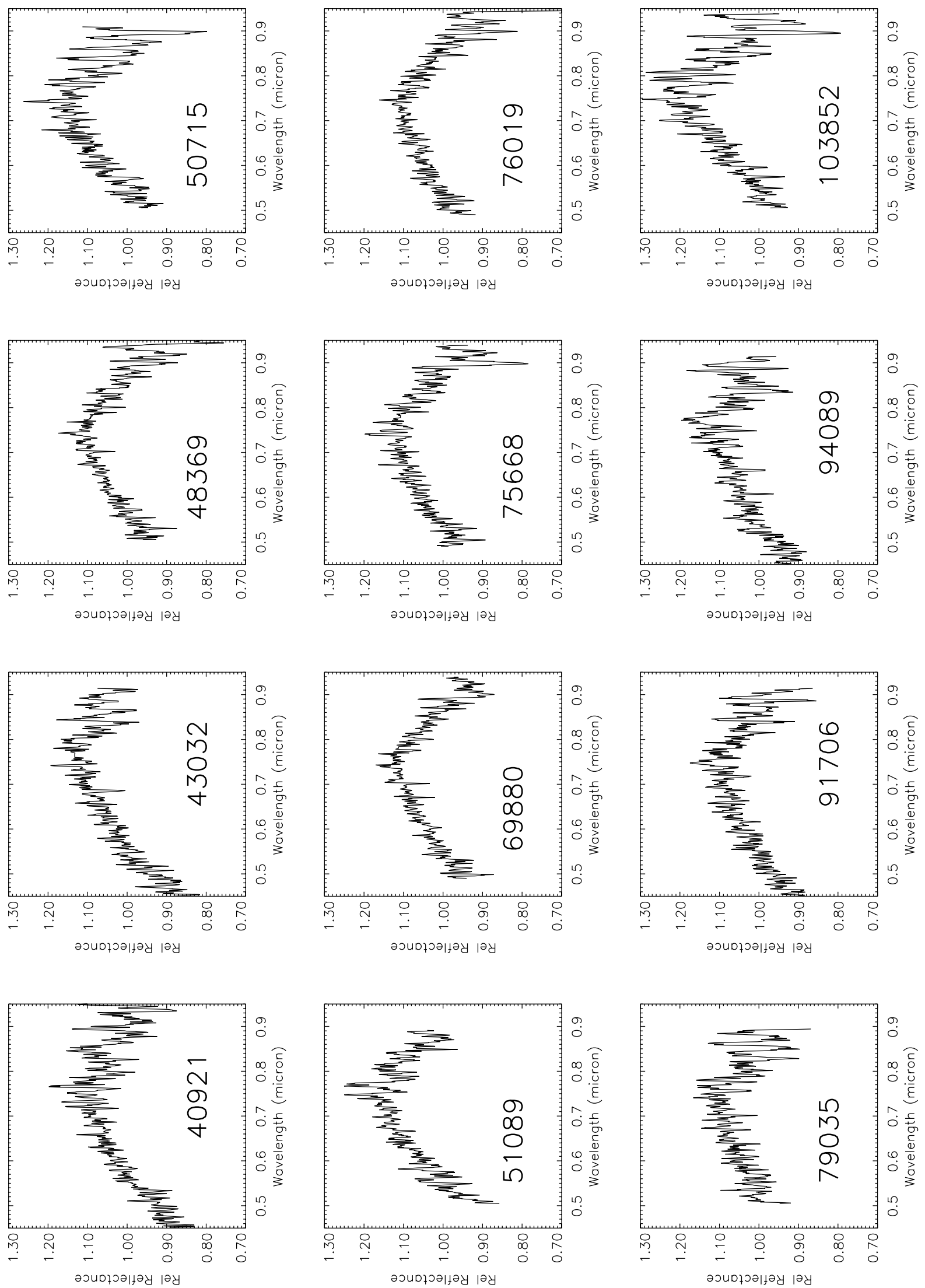
P. Vernazza et al.: Karin family, Online Material p 5
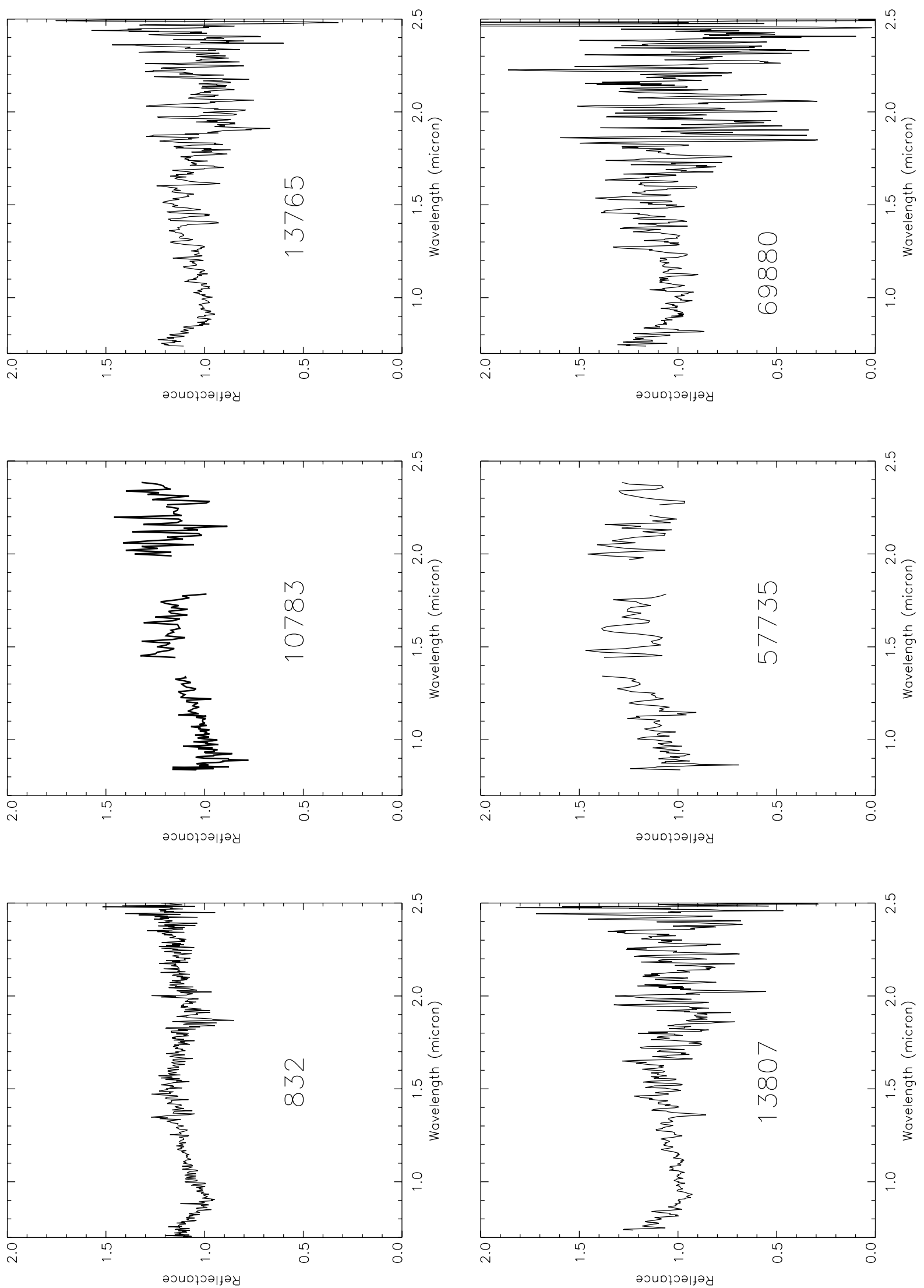